\title{
A Posteriori Error Estimation via Equilibrated Stress Reconstruction for Unilateral Contact Problems
}

\author{
Ilaria Fontana ${ }^{* 1,2}$, Daniele Di Pietro ${ }^{1}$ and Kyrylo Kazymyrenko ${ }^{2}$ \\ ${ }^{1}$ Institut Montpelliérain Alexander Grothendieck (IMAG) \\ University of Montpellier \\ 34090 Montpellier, France \\ 2 EDF Lab Paris-Saclay, ERMES Department \\ 91120 Palaiseau, France
}

\begin{abstract}
Engineering teams often use finite element numerical simulations to study large hydraulic structures. In particular, the models for concrete dams have to be capable to take into account the non-linear behavior of discontinuities which strongly depends on the presence of contact. Furthermore, during simulations based on the finite element approximation, the quality of the numerical solution can be measured through some local a posteriori error estimators which can give information on the local error $[1,6]$. These local estimators can be used to distinguish the different components of the error, to control locally the error and to perform an adaptive refinement of the mesh.

In this presentation we focus on the unilateral contact problem without friction, in which the contact is mathematically represented by some inequalities on the involved boundary part. In literature different numerical methods are proposed, notably penalty methods, mixed methods and Nitschebased methods [4]. We consider the latter since it allows to treat contact boundary conditions in a weak sense with a consistent formulation and without the introduction of additional unknowns such as Lagrange multipliers. For this problem we show a posteriori error estimation based on an equilibrated stress reconstruction. This equilibrated stress is used to compute some local and global estimators which separate the different components of the computational error. In particular, the local estimators show how the error is distributed over the mesh and, as a consequence, provide a method to refine the mesh in an adaptive way. Moreover, based on the properties of these estimator, we can define local or global stopping criteria for the linearization solver and for the adjustment of some parameters. Motivated by the results of $[3,5]$, we also propose a practical way to obtain an equilibrated, $\mathrm{H}$ (div)-conforming, weakly symmetric stress reconstruction via local problems defined on patches around the vertices of the mesh using the Arnold-Falk-Winther mixed element space [2]. Finally, we present some simulations in which we adaptively refine the coarse initial mesh using the distribution of the local estimators and obtaining a better convergence order of the error.
\end{abstract}

\section{REFERENCES}

[1] Ainsworth, M. and Oden, J.T. A Posteriori Error Estimation in Finite Element Analysis. Wiley, 2000.

[2] Arnold, D.N., Falk, R.S and Winther R. Mixed finite element methods for linear elasticity with weakly imposed symmetry. Mathematics of Computation, Vol. 76, pp. 1699-1723, (2007).

[3] Botti, M. and Riedlbeck, R. Equilibrated stress tensor reconstruction and a posteriori error estimation for nonlinear elasticity. Computational Methods in Applied Mathematics, Vol. 20, pp. 39-59, (2020). 
[4] Chouly, F., Fabre, M., Hild, P., Mlika, R., Pousin, J. and Renard, Y. An overview of recent results on Nitsche's method for contact problems. Geometrically Unfitted Finite Element Methods and Applications, Vol. 121, pp. 93-141, (2017).

[5] Riedlbeck, R., Di Pietro, D. and Ern, A. Equilibrated stress tensor reconstruction for linear elasticity problems with application to a posteriori error analysis. Finite Volumes for Complex Applications VIII - Methods and Theoretical Aspects, pp. 293-301, (2017).

[6] Vohralík, M. A posteriori error estimates for efficiency and error control in numerical simulations. UPMC Sorbonne Universités, February 2015.
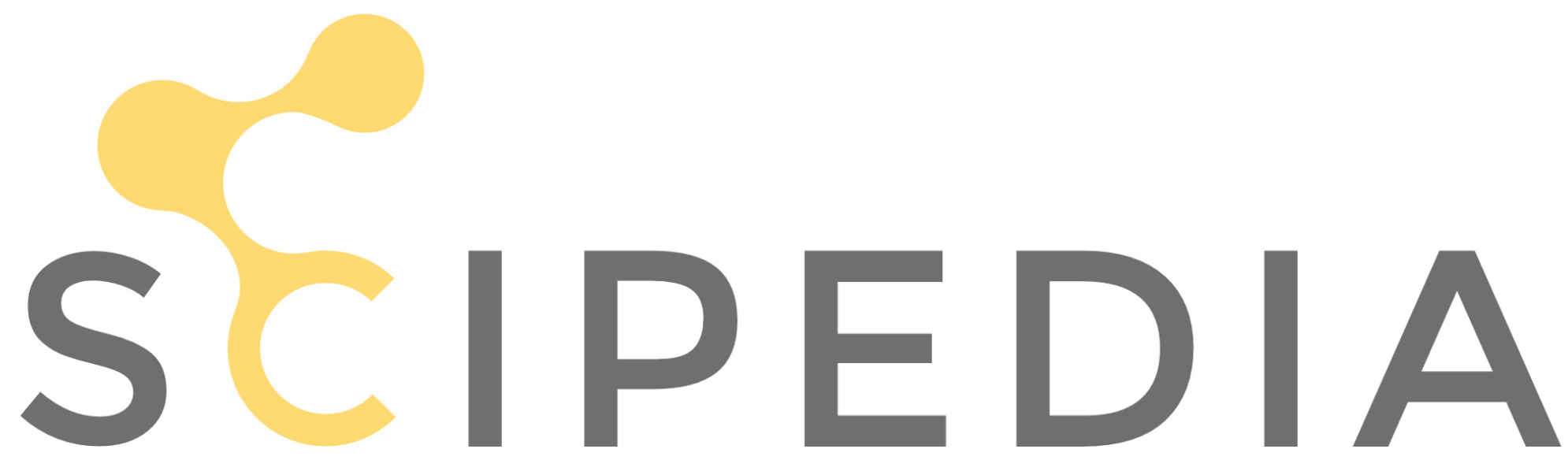

Register for free at https//www.scipedia.com to download the version without the watermark 